the elements are present in equal amount, and no addition of an element to the substance to be analysed needs to be made. The assumption is made that, for an alloy of two elements of nearly equal atomic number, the ratio of the number of the atoms of the two elements present is equal to the ratio of the intensities of corresponding lines (say the $K a_{1}$ lines) in the spectrum of the alloy, provided these lines are excited under equivalent conditions. The X-ray line intensities are determined photographically by photometering the lines, and by determining the $\mathrm{X}$-ray Hurter and Driffield curve of the emulsion used. The determination of line intensities in this way is found to be both convenient and accurate. The assumption mentioned has been verified to a quite satisfactory degree of accuracy by making an X-ray analysis of the following six alloys of known composition :

$\mathrm{Cu} 73$ per cent, $\mathrm{Zn} 27$ per cent; $\mathrm{Cu} 1 \cdot 1, \mathrm{Zn} 98.9$; Cu $0 \cdot 11, \operatorname{Zn} 99 \cdot 89 ; \mathrm{Sn} 71, \mathrm{Cd} 29 ; \mathrm{Pb} 60 \cdot 5, \mathrm{Bi} 39 \cdot 5$; and 12 per cent $\mathrm{Zn}$ in $\mathrm{Zn}$-Sn-Cu.

The alloys used included both heterogeneous and homogeneous structures; and in some cases the $K$ series was used, in others the $L$ series.

Our observations are not in agreement with those of previous workers in respect to the effect of a third element, an effect referred to by Prof. Hevesy. Coster and Nishina found that the presence of a third element in a powder mixture affected the accuracy of their results in cases when no selective absorption took place, and they concluded that the same method as we have used was not accurate. For alloys, however, we find it quite accurate.

Natural Philosophy Laboratory,

T. H. LABY.

C. E. EDDY.

University of Melbourne, Feb. 5.

\section{Nature of the Magnetisation Curve of Single Iron Grystals.}

IN recent years various observers have investigated the magnetisation of single iron crystals. Their results differ in several important respects. From investigations of iron crystals made in these laboratories, we believe that the observed differences are ascribable mainly to the unsatisfactory accuracy with which magnetic field intensity has been estimated when the demagnetisation factor is very large, or when it is a function of the magnetisation, as it is in specimens that are not ellipsoids. In the latter case, the only way in which the field intensity can be determined is by direct measurement. Experimenters, strangely enough, have seldom employed this procedure, preferring rather to rely on calculation of the demagnetising component of the field intensity by methods which have been shown to rest on invalid assumptions.

The outstanding problem evoked by these discrepant observations is the question of whether or not there are 'breaks' (abrupt changes of slope) in the magnetisation curves and hysteresis loops. The existence of this phenomenon was announced by W. Gerlach (Phys. Zeits., 26, $914 ; 1925$ ) four years ago. Since then its reality has been repeatedly confirmed or denied by other observers.

We believe that the breaks appearing in these curves are spurious; and that in the few cases where it is not possible to draw a smooth curve fitting the points equally well, they are due to incorrect estimation of the field intensities. In support of this idea we offer the following facts :

When a sufficient number of points on the curve are obtained and the actual magnetising field-the applied field less the demagnetising component-is measured with considerable accuracy, the magnetisation curve has no breaks (Foster, Phys. Rev., 33, 1071 ; 1929). If, however, these same data are plotted without applying the measured field intensity correc. tions, curves are obtained which in most cases are so flattened at the knee that it is possible to construct that portion of the curve with one or two sharp changes of slope. The reason for this is that the demagnetising component of the field intensity usually goes through a rather sharp maximum in the same range of magnetisation as is occupied by the knee of the curve. This maximum is always present in uniform cylinders. In iron crystals the curve of demagnetising component vs. magnetisation, or ' correction curve', is unlike that for ordinary iron, and is similar in shape to that obtained in polycrystalline permalloy (Foster, Phil. Mag., 8, 312 ;

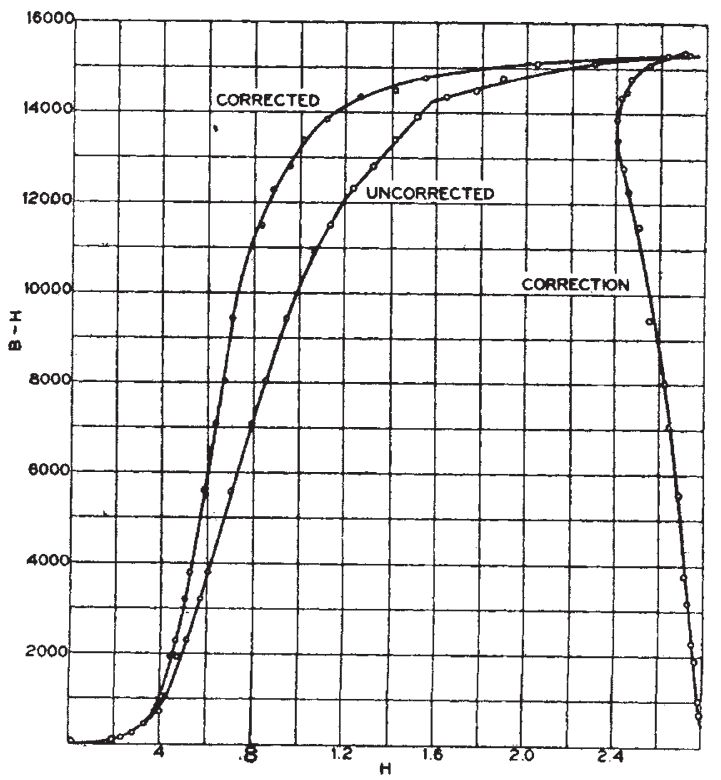

FIQ. 1.-Magnetisation curve of an iron crystal showing the characteristic correction curve and the effect of omitting it.

1929). In no case is this correction curve the familiar straight line ("shearing curve') which has been so generally assumed.

There have been three other researches in which the intensity of the actual magnetising field was measured. In two cases (Dussler and Gerlach, Zeits. f. Physik, 44, 279; 1927; Gries and Esser, Archiv. f. Elektrotech., 22, 145; 1929) the authors show curves with breaks; but there are not enough points in the region in question to justify them. In the third (Wolman, Archiv. f. Elektrotech., 19, 385; 1928) the published curves are smooth. Sizoo (Zeits. f. Physik, $56,649 ; 1929)$, whose specimens were cylinders with rounded ends, regards the fact that his uncorrected curve is broken as proof that the true curve must be regarded as a broken one. Our determination of the actual field corrections for cylindrical iron crystals indicates that only the uncorrected curve is flattened at the knee. The explanation of the breaks is illustrated in Fig. 1.

Donald Foster.

RICHARD M. BOZORTh.

Bell Telephone Laboratories,

New York, N.Y. Jan. 15 .

No. 3153 , VoL. 125] 